\title{
Isolated intradural Rosai-Dorfman disease of the spine: report of two cases
}

\author{
Yu Xin ${ }^{1 \dagger}$, Hui Shen ${ }^{2 \dagger}$, Desheng Kong ${ }^{1}$, Wenqing Jia ${ }^{1}$, Hai Liu ${ }^{1}$ and Jun Yang ${ }^{1 *}$
}

\begin{abstract}
Background: Rosai-Dorfman disease (RDD) is an idiopathic histiocytic proliferative disorder characterized by sinus histiocytosis and massive lymphadenopathy. Extranodal and central nervous system (CNS) involvement is unusual. We describe two distinctly rare cases of isolated intradural RDD of the spine without cervical lymphadenopathy.

Case presentation: We report our experience in the management of two patients with RDD. The first patient had two ventral intradural lesions, radiologically in favour of multiple spinal meningiomas, and the second patient presented an intradural extramedually lesion, extending into the right foramina, suggestive of a sacral perineural cyst. Treatments consisted of total excision and subtotal excision without steroids and radiotherapy, respectively. The definitive diagnosis was confirmed by histopathology and immunochemistry.
\end{abstract}

Conclusions: RDD should be considered in the rare differential diagnosis of a spinal disease. Resection of the lesions is an effective treatment choice to relieve spinal compression symptoms. The efficacy of the adjuvant therapy to control the disease is unknown.

Keywords: Rosai-Dorfman disease, Sinus histiocytosis with massive lymphadenopathy, Meningiomas, Spinal cord

\section{Background}

Rosai-Dorfman disease (RDD) is an idiopathic histiocytic proliferative disorder characterized by sinus histiocytosis with massive lymphadenopathy [1]. Typical clinical presentation involves massive painless cervical lymphadenopathy, but extranodal disease is apparent in about $40 \%$ of cases, most commonly involving the orbit, skin, respiratory tract, bones, kidneys, heart, and head and neck region $[1,2]$. In less than $5 \%$ of the cases, the central nervous system (CNS) is involved, with three fourths in brain and one fourth in spinal cord [3]. We describe two cases of isolated intradural RDD of the spine, and briefly review the literature.

\section{Case presentation}

Case 1

A 40-year-old man presented with a 6-month history of neck back pain and a 4-week history of bilateral lower-

\footnotetext{
* Correspondence: 13911707098@163.com

${ }^{\dagger}$ Equal contributors

'Department of Neurosurgery, Beijing Tiantan Hospital, Capital Medical University, No. 6, Tiantan Xili, Dongcheng District, Beijing 100050, People's Republic of China

Full list of author information is available at the end of the article
}

limb weakness. Neurological examination revealed a paraparesis (muscle power of grade 4/5) without a bilateral Babinski sign. General examination was unremarkable and the patient had no clinical evidence of lymphadenopathy.

A magnetic resonance image (MRI) showed 2 distinct ventral intradural lesions with similar characteristics, homogeneously isointense on T1-weighted as well as on T2-weighted images, with marked homogeneous enhancement after gadolinium administration. The extent of lesion was from C3 to C5 $(3.3 \mathrm{~cm} \times 1.2 \mathrm{~cm}$, and C6 to C7 $(0.8 \mathrm{~cm} \times 0.9 \mathrm{~cm})$. A dural tail was observed at the ventral dura (Fig. 1a-d). The patient's chest x-ray was normal, and his white blood cell count, sedimentation rate and $\mathrm{C}$ reactive protein were within normal limits.

A C3-C6 laminectomy was performed, allowing complete resection of both intradural lesions without manipulation of the spinal cord. The lesions were pinkishgray, and encasing the ventral dura. Histological study of the lesions revealed large lymphoplasmacytic areas embedded with sheets of multinucleated histiocytic cells. The histiocytic cells showed evidence of emperipolesis, CD68 and S100 immunopositivity, and negative labeling for 


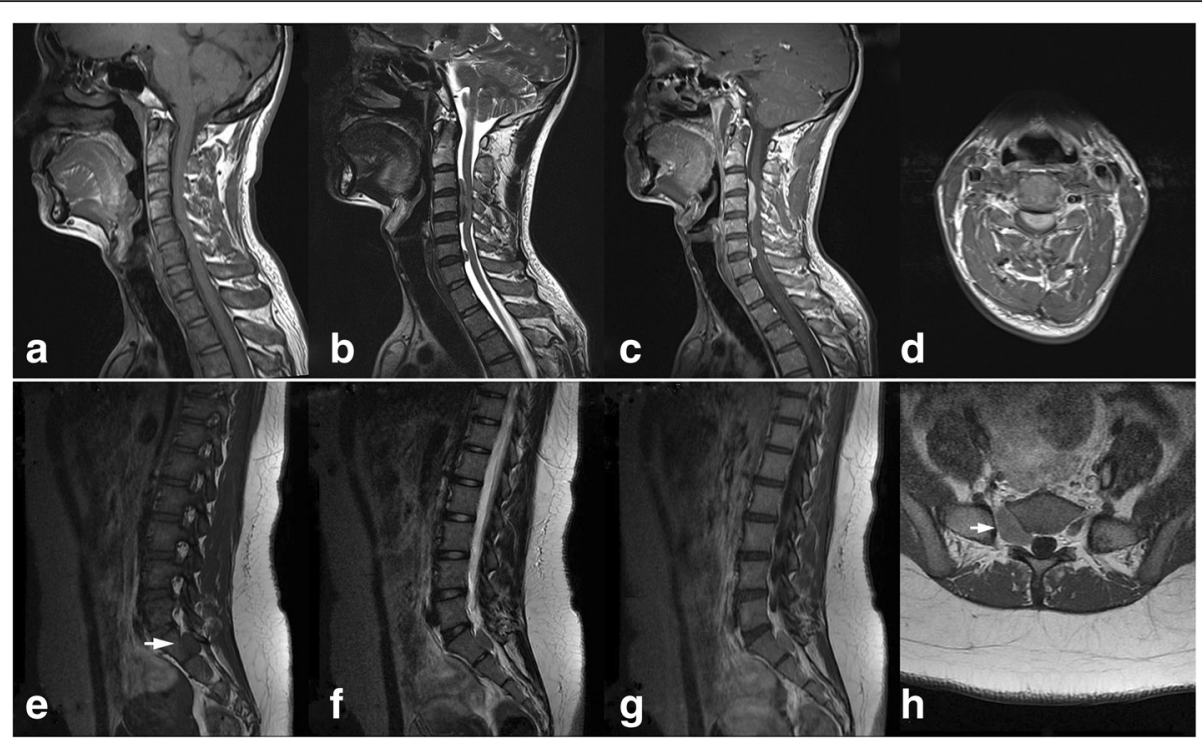

Fig. 1 Case 1. A preoperative MRI showing 2 distinct ventral, intradural and extramedullary lesions with homogeneously isointenseon both sagittal T1- and T2-weighted images ( $\mathbf{a}$ and $\mathbf{b}$ ), with marked homogeneous enhancement on the sagittal (c) and axial (d) after gadolinium administration. A dural tail was observed at the ventral dura. Case 2.A preoperative MRI showing a S1/2 level extramedullary lesion with similar isointenseon both sagittal T1- and T2-weighted images (e and $\mathbf{f}$ ), and slight heterogeneous enhancement on the sagittal (g) and axial (h) after gadolinium administration

CD1a. These characteristics were compatible with RosaiDorfman disease.

Since a complete resection of the mass was performed and lymphadenopathy was not observed, radiation and chemotherapy were not performed. The patient has been followed up for 2 years with no new complaints, and with improving neurologic deficits.

\section{Case 2}

A 14-year-old female presented to our outpatient clinic with a 4-month history of intermittent pain in her both lower extremities. No bowel or bladder symptoms had been reported. She had hyporeflexia in the right lower limb. The rest of her neurological and systemic examinations were unremarkable. Routine hematologic tests were within reference range.

Preoperative MRI images of the patient's lumbosacral spine indicated intradural lesion (about $2.5 \mathrm{~cm} \times 2.2 \mathrm{~cm}$ ), extending into the right foramina at the $\mathrm{S} 1 / 2$ level. This lesion presented with similar isointense on both T1- and T2-weighted MR images, with marked slight heterogeneous enhancement after gadolinium administration (Fig. 1e-h). Routine hematologic and biochemical tests were within reference range. Computed tomography of the chest and abdomen showed no significant lymphadenopathy. It was provisionally diagnosed as a sacral perineural cyst.

The patient underwent a S1-2 laminectomy to reveal a firm pink-gray intradural lesion extending into the right foramina at the S1/2 level. The lesion encased and compressed the right S1 nerve root. Based on the need to protect the patient's neurological functions, the lesion was partially removed under spinal evoked-potential monitoring. A histopathological examination revealed that aggregates of pale-staining foamy histiocytes phagocytosed lymphocytes and plasma cells in a background of collagen and reticulin fiber. Immunohistochemistry revealed that the lesional histiocytes were positive for CD68, and S-100 but negative for CD1a by immunohistochemistry, distinguishing this disorder from Langerhans cell histiocytosis. Thus confirming the diagnosis of RDD (Fig. 2a-c).

The patient had an uneventful postoperative course. At 2-month post-operation, follow-up physical examination disclosed that her lower extremity pain had gradually disappeared and reflexes were normal. Subsequently regular clinical and imaging follow-up over the next 1 years showed no signs or symptoms of recurrence.

\section{Discussion}

Sinus histiocytosis with massive lymphadenopathy was first described by Rosai and Dorfman in 1969 [2]. The etiology of RDD remains unknown. RDD is hypothesized to occur secondary to an abnormal immunological response or an infectious factor. Recent studies suggested that infective agents like HHV-6 and Parvo B19 might be the causative factors [4]. RDD is characterized by massive, painless cervical lymphadenopathy variably associated with fever, leukocytosis, elevated erythrocyte sedimentation rate and weight loss. RDD of the nervous system has also been reported, and most cases involve the brain [5]. Spinal cord involvement such as intramedullary, intradural and extradural is apparently very rare 


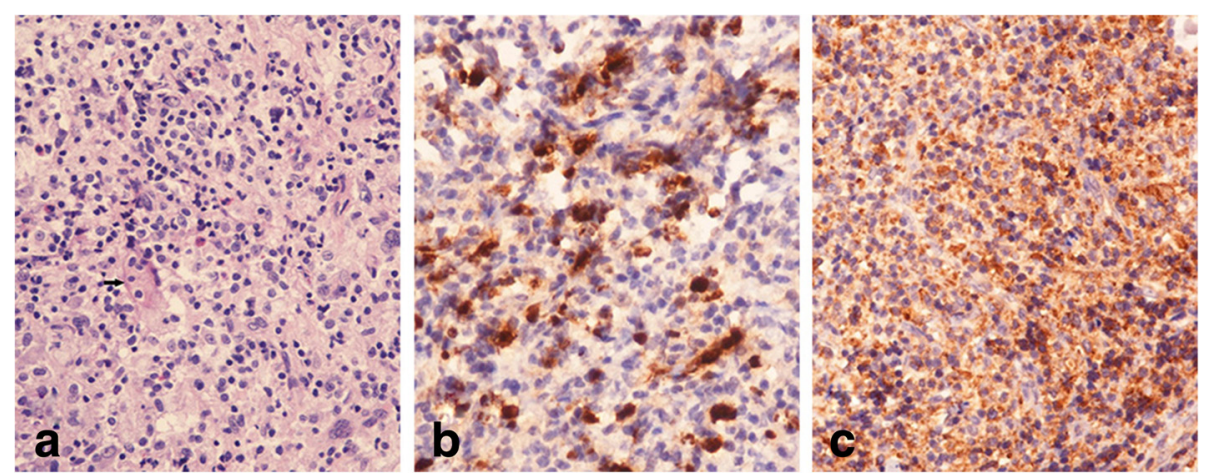

Fig. 2 Histopathology shows a lesion composed of dilated spaces filled with histiocytes admixed with lymphocytes and plasma cells (a: hematoxyeosin stain, Original magnification $\times 200$ ). The histiocytic cells show strong immunoreactivity for S100 and CD68 (b, c: Original magnification × 200)

in RDD [6-10]. Over $90 \%$ of CNS RDD involves the leptomeninges. In neuroimaging it is seen as a duralbased, contrast-enhancing lesion. Thus, clinically and radiologically, the disease is thought to represent meningioma. In the first case, the lesions were confined to the intradural extramedullary of the cervical spine, which were provisionally diagnosed as multiple spinal meningiomas [7-9]. As in the present of the second case, isolated intradural RDD of the lumbosacral spine, extending into the right foramina at the S1/2 level is extremely rare.

The MRI features of isolated RDD were variable and could be misleading. In most cases, as in the present patients, the signals of the lesion ranged from iso- to hypointensity on $\mathrm{T} 1$ weighted images, and iso- to hyperintensity on $\mathrm{T} 2$ weighted images, and exhibits an intense and uniform enhancement after gadolinium administration $[1,3,6-8,10-13]$. MR images are usually indistinguishable from other more common lesions in the extradural spinal space such as meningioma [7, 9, 14], lymphoma, and sarcoma [6]. RDD displayed neuroimaging features typical of meningioma with a dural tail. There are no pathognomonic radiographic marks to suggest a particular preoperative diagnosis. Therefore, the definitive diagnosis of RDD relies on histopathological examination, and the immunochemical analyses contribute to determining the final diagnosis [15].

The characteristic histopathological pattern of RDD is emperipolesis, which refers to phagocytosis of intact lymphocytes by macrophages. Large histiocytes of RDD are immunoreactive for S100 protein and CD68, but not for CD1a. This feature helps to distinguish from Langerhans cell histiocytosis, which is positive for CD1a [16].

The natural history of RDD has been reported to be benign with spontaneous remission. In some patients, however, rapid disease progression, multisystem involvement, and even fatal outcomes have been described [1, 2]. In case of persistent or progressive disease, a number of adjuvant therapies have been administered with variable success, including radiotherapy and chemotherapy with corticosteroids, 6-mercaptopurine and methotrexate [17]. Administration of steroids often resolve fever and reduce lymph node size. Radiation or chemotherapy has been shown to be beneficial in achieving complete remission in $33.3 \%$ or $16.6 \%$ of cases [18]. Resection of the lesions is an effective treatment choice to relieve spinal compression symptoms. There is no established systemic treatment protocol for RDD, since it is a rare disease presenting with different clinical features. Because the patients had improved neurological course after surgery, adjuvant treatments with chemotherapy or radiotherapy are unnecessary until disease progression is detected.

\section{Conclusions}

In summary, we present two cases of isolated intradural RDD of the spine. It should be considered among the rare differential diagnosis of intradural cord compression. Resection of the lesions is an effective treatment choice to relieve spinal compression symptoms. The efficacy of the adjuvant therapy to control the disease is unknown.

\section{Consent}

Written informed consent was obtained from the patient for publication of this case report and any accompanying images. A copy of the written consent is available for review by the Editor-in-Chief of this journal.

\section{Abbreviations \\ CNS: Central nervous system; MRI: Magnetic resonance image; RDD: Rosai- Dorfman disease.}

\section{Competing interests}

The authors declare that they have no competing interests.

\section{Authors' contributions \\ YX and HS collected the patient data and drafted the manuscript. DK and WJ participated in surgical treatment and design of the study. $\mathrm{HL}$ participated in the design of the study and assisted with revisions of the manuscript. JY conceived of this study and participated in its design and coordination and helped to draft the manuscript. All authors read and approved the final manuscript.}




\section{Acknowledgements}

The authors have no personal financial or institutional interest in any of the drugs, materials, or devices described in this article.

\section{Author details}

'Department of Neurosurgery, Beijing Tiantan Hospital, Capital Medical University, No. 6, Tiantan Xili, Dongcheng District, Beijing 100050, People's Republic of China. ${ }^{2}$ Department of Neurosurgery, Beijing Pinggu Hospital, No.59, Xinping North Road, Pinggu District, Beijing, People's Republic of China.

Received: 21 October 2015 Accepted: 3 May 2016

\section{Published online: 18 May 2016}

\section{References}

1. Foucar E, Rosai J, Dorfman R. Sinus histiocytosis with massive lymphadenopathy (Rosai-Dorfman disease): review of the entity[J]. Semin Diagn Pathol. 1990;7(1):19-73.

2. Rosai J, Dorfman RF. Sinus histiocytosis with massive lymphadenopathy. A newly recognized benign clinicopathological entity[J]. Arch Pathol. 1969;87(1):63-70.

3. Hollowell JP, Wolfla CE, Shah NC, et al. Rosai-Dorman disease causing cervical myelopathy[J]. Spine (Phila Pa 1976). 2000;25(11):1453-6.

4. Mehraein $Y$, Wagner $M$, Remberger $K$, et al. Parvovirus B19 detected in Rosai-Dorfman disease in nodal and extranodal manifestations[J]. J Clin Pathol. 2006;59(12):1320-6.

5. Kattner KA, Stroink AR, Roth TC, et al. Rosai-Dorfman disease mimicking parasagittal meningioma: case presentation and review of literature[J]. Surg Neurol. 2000;53(5):452-7, 457.

6. Osenbach RK. Isolated extranodal sinus histiocytosis presenting as an intramedullary spinal cord tumor with paraplegia. Case report[]]. J Neurosurg. 1996;85(4):692-6.

7. Hargett C, Bassett T. Atypical presentation of sinus histiocytosis with massive lymphadenopathy as an epidural spinal cord tumor: a case presentation and literature review[J]. J Spinal Disord Tech. 2005;18(2):193-6.

8. Al-Saad K, Thorner P, Ngan BY, et al. Extranodal Rosai-Dorfman disease with multifocal bone and epidural involvement causing recurrent spinal cord compression[J]. Pediatr Dev Pathol. 2005;8(5):593-8.

9. Bhandari A, Patel PR, Patel MP. Extranodal Rosai-Dorfman disease with multiple spinal lesions: a rare presentation[J]. Surg Neurol. 2006;65(3):308-11.

10. Maiti TK, Gangadharan J, Mahadevan A, et al. Rosai-Dorfman disease presenting as cervical extradural lesion: a case report with review of literature[J]. Neurol India. 2011;59(3):438-42.

11. Andriko JA, Morrison A, Colegial CH, et al. Rosai-Dorfman disease isolated to the central nervous system: a report of 11 cases[J]. Mod Pathol. 2001;14(3):172-8.

12. Kidd DP, Revesz T, Miller NR. Rosai-Dorfman disease presenting with widespread intracranial and spinal cord involvement[J]. Neurology. 2006;67(9):1551-5.

13. Abou-Zeid AH, Herwadkar A, du Plessis D, et al. Isolated extradural RosaiDorfman disease of the thoracic spine: a rare cause of spinal cord compression: case report[J]. Neurosurgery. 2010;67(2):E514-5.

14. Ma J, Xiao J, Wang L. Extranodal Rosai-Dorfman disease with multilevel lumbar spinal lesions[J]. J Neurosurg Spine. 2008;9(1):55-7.

15. Roy C, Saha A, Roy S, et al. Extranodal Rosai-Dorfman Disease presenting as spinal extradural lesion: a case report with a review of the literature[J]. J Cancer Res Ther. 2012;8(4):647-9.

16. Dran G, Rasendrarijao D, Vandenbos F, et al. Rosai-Dorfman disease causing spinal cord compression: case report[J]. Neurosurgery. 2008;62(4):E977-8, E978.

17. Tubbs RS, Kelly DR, Mroczek-Musulman EC, et al. Spinal cord compression as a result of Rosai-Dorfman disease of the upper cervical spine in a child[J]. Childs Nerv Syst. 2005;21(11):951-4.

18. Pulsoni A, Anghel G, Falcucci P, et al. Treatment of sinus histiocytosis with massive lymphadenopathy (Rosai-Dorfman disease): report of a case and literature review[J]. Am J Hematol. 2002;69(1):67-71.

\section{Submit your next manuscript to BioMed Central and we will help you at every step:}

- We accept pre-submission inquiries

- Our selector tool helps you to find the most relevant journal

- We provide round the clock customer support

- Convenient online submission

- Thorough peer review

- Inclusion in PubMed and all major indexing services

- Maximum visibility for your research

Submit your manuscript at www.biomedcentral.com/submit 